\title{
Revalidación de Aristolochia pearcei Phil. y notas acerca de la identidad de A. bridgesii (Klotzsch) Duch. (Aristolochiaceae)
}

\section{Revalidation of Aristolochia pearcei Phil. and notes on the identity of $A$. bridgesii (Klotzsch) Duch. (Aristolochiaceae)}

\author{
Patricio Saldivia Pérez ${ }^{1 *}$ \& Luis Faúndez Yancas ${ }^{1,2}$
}

${ }^{1}$ BIOTA, Gestión y Consultorías Ambientales Limitada, Miguel Claro 1224, Providencia, Santiago, Chile. ${ }^{2}$ Herbario AGUCH, Facultad de Ciencias Agronómicas, Universidad de Chile, Santa Rosa 11315, La Pintana, Santiago, Chile. *patricio.saldivia.perez@gmail.com

\begin{abstract}
RESUMEN
Mediante un estudio detallado de las descripciones originales y de los ejemplares tipo, además del análisis de nuevos materiales recolectados, se establece que Aristolochia bridgesii y A. pearcei, entidades hasta ahora consideradas sinónimos, corresponden a especies distintas, con una morfología floral notoriamente diferente y con áreas de distribución alopátricas separadas por más de $300 \mathrm{~km}$ en sentido latitudinal. De este modo, para Chile se reconocen, a la fecha, tres especies, $A$. bridgesii, A. pearcei y A. chilensis. Se entrega una completa ilustración de $A$. bridgesii y una clave para el género en Chile. Adicionalmente $A$. bridgesii y $A$. pearcei son lectotipificadas.
\end{abstract}

Palabras clave: Aristolochia bridgesii, material tipo, lectotipificación, Chile, endemismo.

\begin{abstract}
Through a detailed study of the original descriptions and type materials, in addition to analyses of the new collected material, we have established that Aristolochia bridgesii and A. pearcei, entities that have been considered synonyms until now, correspond to different species, with notoriously different floral morphology and allopatric geographic distributions, separated in latitude by more than $300 \mathrm{~km}$. Thus, three species are recognized for Chile: $A$. bridgesii, $A$. pearcei and $A$. chilensis. A complete illustration of $A$. bridgesii and key for the genus in Chile are provided. In addition, A. bridgesii and A. pearcei are lectotypified.
\end{abstract}

KEYwords: Aristolochia bridgesii, type material, lectotypification, Chile, endemism.

\section{INTRODUCCIÓN}

En Chile la familia Aristolochiaceae cuenta sólo con el género Aristolochia para el cual se reconocen dos especies (Marticorena \& Quezada 1985, Ruiz, 2001, Zuloaga et al. 2008), Aristolochia chilensis Bridges ex Lindl. para la costa y depresión intermedia entre las regiones Metropolitana y de Atacama, y Aristolochia bridgesii (Klotzsch) Duch. para la precordillera de las provincias de Limarí y Elqui en la Región de Coquimbo y provincia del Huasco en la Región de Atacama (Ruiz, 2001).

No obstante, la actual distribución asignada para $A$. bridgesii (regiones de Coquimbo y Atacama) (Ruiz 2001, Zuloaga et al. 2008) es motivo de confusión, ya que fue descrita originalmente para "Colchagua" (Región de
O’Higgins), $400 \mathrm{~km}$ al sur de donde actualmente se asume su distribución.

Por su parte, A. pearcei Phil., descrita en 1861, no fue considerada como especie válida para la flora de Chile (Marticorena \& Quezada 1985), siendo luego incluida en la sinonimia de Aristolochia bridgesii (Klotzsch) Duch. (Ruiz 2001) sin mencionar los argumentos para ello y al igual que para esta última, su distribución resulta controversial ya que originalmente fue descrita para las cercanías de Chillán (Región del Bío-Bío), $600 \mathrm{~km}$ al sur de donde actualmente se asume su distribución.

La presente nota tiene por objetivo validar a $A$. pearcei como especie distinta de $A$. bridgesii y aclarar la distribución de ambas, sobre la base de antecedentes bibliográficos, revisión de materiales tipo y nuevas recolecciones. 


\section{MATERIALES Y MÉTODOS}

Se revisaron descripciones originales y material tipo de Aristolochia pearcei en SGO y de Howardia bridgesii Klotzsch en $\mathrm{K}$ y $\mathrm{BM}$, los de ésta última por medio de la plataforma Global Plant Initiative (plants.jstor.org). Estos fueron comparados con materiales recolectados por los autores y otros recolectores entre 1976 y 2010 en las regiones de O'Higgins y Metropolitana y trabajos de campo realizados en las regiones de Coquimbo y Atacama. Materiales de A. bridgesii fueron depositados en los herbarios del Museo Nacional de Historia Natural (SGO), de la Universidad de Concepción (CONC) y de la Facultad de Ciencias Agronómicas de la Universidad de Chile (AGUCH).

\section{RESULTADOS Y DISCUSIÓN}

\section{ReValidación de ARISTOLOCHIA PEARCEI}

A. pearcei fue descrita por Rodulfo Philippi en 1861 sobre la base de una muestra colectada por Ricardo Pearce durante su estadía en el centro sur de Chile en 1860, supuestamente en las cercanías de Chillán como se indica en el protólogo. No obstante, el mismo autor en una segunda descripción de A. pearcei (Philippi 1865), señala con dudas la localidad de Chillán como referencia al origen de la especie "Specimen prope Chillan?", y posteriormente (Philippi 1895) indica que la especie fue erróneamente señalada para Chillán, siendo propia del Valle del Huasco, cerca de Yerbas Buenas.

Este nombre no ha sido reconocido dentro de las especies aceptadas para Chile, probablemente por ser considerado dentro de la sinonimia de $A$. bridgesii en el Catálogo de la Flora Vascular de Chile (Marticorena \& Quezada, 1985), ya que sólo se mencionan $A$. chilensis y A. bridgesii, lo que es ratificado por Marticorena (1990) y por Ruiz (2001), siendo este último quien realiza explícitamente la sinonimia, aunque sin señalar argumento alguno.

Se revisó el material original de $A$. pearcei depositado en el Museo Nacional de Historia Natural (SGO 47944), el cual concuerda con grupos poblacionales distribuidos en el norte de la Región de Coquimbo y sur de la Región de Atacama, principalmente en la forma y tamaño del labio, y en el color y completa pubescencia interna del perigonio (Fig 1). De esta forma, Aristolochia pearcei corresponde a la especie señalada bajo el nombre $A$. bridgesii para la flora de Chile (Ruiz 2001), siendo A. bridgesii en realidad una especie distribuida más al sur, en las regiones Metropolitana, de O'Higgins y probablemente del Maule (ver Notas acerca de $A$. bridgesii).

R. Philippi no señala material tipo, sin embargo indica que se basó en el material recolectado por R. Pearce. Muñoz-Pizarro (1960) indica dicha colección, por lo que se designa como lectotipo (ver Consideraciones taxonómicas más adelante). La Figura 1 muestra la correspondencia del material tipo con la morfología de grupos de poblaciones ubicadas en el ámbito de la distribución geográfica antes señalada; las coincidencias son evidentes pese a que el material original muestra flores sin un completo desarrollo y en la fotografía no se alcanza a distinguir claramente la pubescencia interna completa del labio.

\section{NotAS ACERCA DE ARISTOLOCHIA BRIDGESII}

Howardia bridgesii fue descrita por Klotzsch en 1859 sobre la base de material colectado por Thomas C. Bridges en Colchagua, Chile, actualmente Región de O'Higgins, y luego transferida al género Aristolochia por Duchartre en 1864. Sin embargo, al parecer lo que en el tiempo de Bridges se reconocía como Colchagua, corresponde actualmente a la zona de Curicó, en la porción norte de la Región del Maule, probablemente dentro de la cuenca media del río Teno, al sur de la Región de O'Higgins (Johnston 1928). Thomas Bridges realizó numerosas colecciones en Chile entre los años 1828 y 1844, situándose en Colchagua (Curicó) entre los años 1834 y 1841 , siendo probablemente las colecciones referidas a este último año (Johnston 1928).

Luego de su descripción, esta especie ha sido escasamente recolectada. En el Museo Nacional de Historia Natural (SGO) no existen materiales y en el herbario de la Universidad de Concepción (CONC) se encuentra depositado un material de Aristolochia bridgesii (CONC 88775) proveniente de San Francisco de Aculeo.

Mediante la revisión de descripciones originales de Howardia bridgesii (Klotzsch 1859) y Aristolochia bridgesii (Duchartre 1864), más la consulta de materiales de las colecciones originales de T. Bridges, depositadas en los herbarios del Royal Botanic Gardens, Kew (K) y Natural History Museum (BM), se ha podido confirmar que concuerdan con el material depositado en el herbario de la Universidad de Concepción (CONC 88775) y con nuevas colecciones realizadas en las regiones de O'Higgins y Metropolitana. Por lo tanto, A. bridgesii no se distribuye en las regiones de Coquimbo y Atacama, siendo propia de las regiones de O'Higgins y Metropolitana, y probablemente en la Región del Maule.

J. Klotzsch no indica material tipo, aunque hace referencia a Bridges junto a la denominación de "Chile", siendo Duchartre quien señala "Colchagua" como localidad típica. T. Bridges enviaba sus materiales a los herbarios Kew (K) y Natural History Museum (BM) (Johnston, 1928), los que aún se encuentran preservados, siendo el depositado en $\mathrm{K}$ el que mantiene la escritura original indicando la localidad referida, por lo que se designa como lectotipo (ver Consideraciones taxonómicas más adelante). Existen otros dos materiales originales de T. Bridges depositados en BM, sin embargo, el etiquetado no es claro como para asumirlos como isolectotipos. BM 993831 indica el año 1898, posterior al fallecimiento de Bridges en 1865, y BM 617504 señala 
el año 1844, cuando Bridges recolectó en Antofagasta y Bolivia (Johnston, 1928). Cabe señalar que para muchas de las colecciones de T. Bridges, la información geográfica es muy escasa, poco clara o incorrecta (Johnston, 1928).

\section{CONSIDERACIONES TAXONÓMICAS}

A continuación se presenta a ambas especies y se detallan algunas de sus principales características morfológicas, su distribución geográfica y ambiental y se entrega una clave para diferenciar las especies del género presentes en Chile, hasta la fecha.

Aristolochia pearcei Phil., Anales Univ. Chile 18:68. 1861. TIPO: R. Pearce s/n (lectotipo SGO 47944! aquí designado) non $A$. pearcei Mast. (1875, hom. illeg. $=A$. weberbaueri O.C. Schmidt). Icon.: Ruiz, 2001, p: 37 fig 7a, bajo el nombre $A$. bridgesii. Figura 1.

Hierba perenne, hemicriptófita, con ramas procumbentes surgiendo desde un xilopodio, herbáceas a semiherbáceas en la base, estriadas, glabras. Hojas ampliamente reniformes, con lóbulos basales divergentes, concurrentes al pecíolo, más anchas que largas, de 3-7 x 4-8 cm, de superficie ondulada, ambas caras de la lámina pubérulas. Flores axilares, solitarias, con utrículo y tubo infundibuliforme de color exterior verde a marrón violáceo; labio amarillo a marrón oscuro, glabro exteriormente y cubierto por pelos blancos en el interior, oblicuo respecto al tubo, ovado a lanceolado, con el extremo distal levemente recurvo. Ginostemio 6-lobado, de $5 \mathrm{~mm}$ de longitud, anteras de 2,5-2,8 mm de longitud, lóbulos del ginostemio de 2-2,3 $\mathrm{mm}$ de longitud y ápices adelgazados y bordes basales involutos, ápices algo incurvos. Ovario ínfero, inmerso en el hipanto, pubérulo con 6 nervios prominentes, levemente contorneado. Fruto una pseudocápsula de 4-5,5, cm de largo. Semillas ligeramente convexas y negruzcas en su parte dorsal, cóncavas de color claro en la ventral, obcordadotriangulares, de aproximadamente 4,5 x $4 \mathrm{~mm}$, cubiertas por prominencias granulares pequeñas de color claro, con la nervadura central ventral prominente.

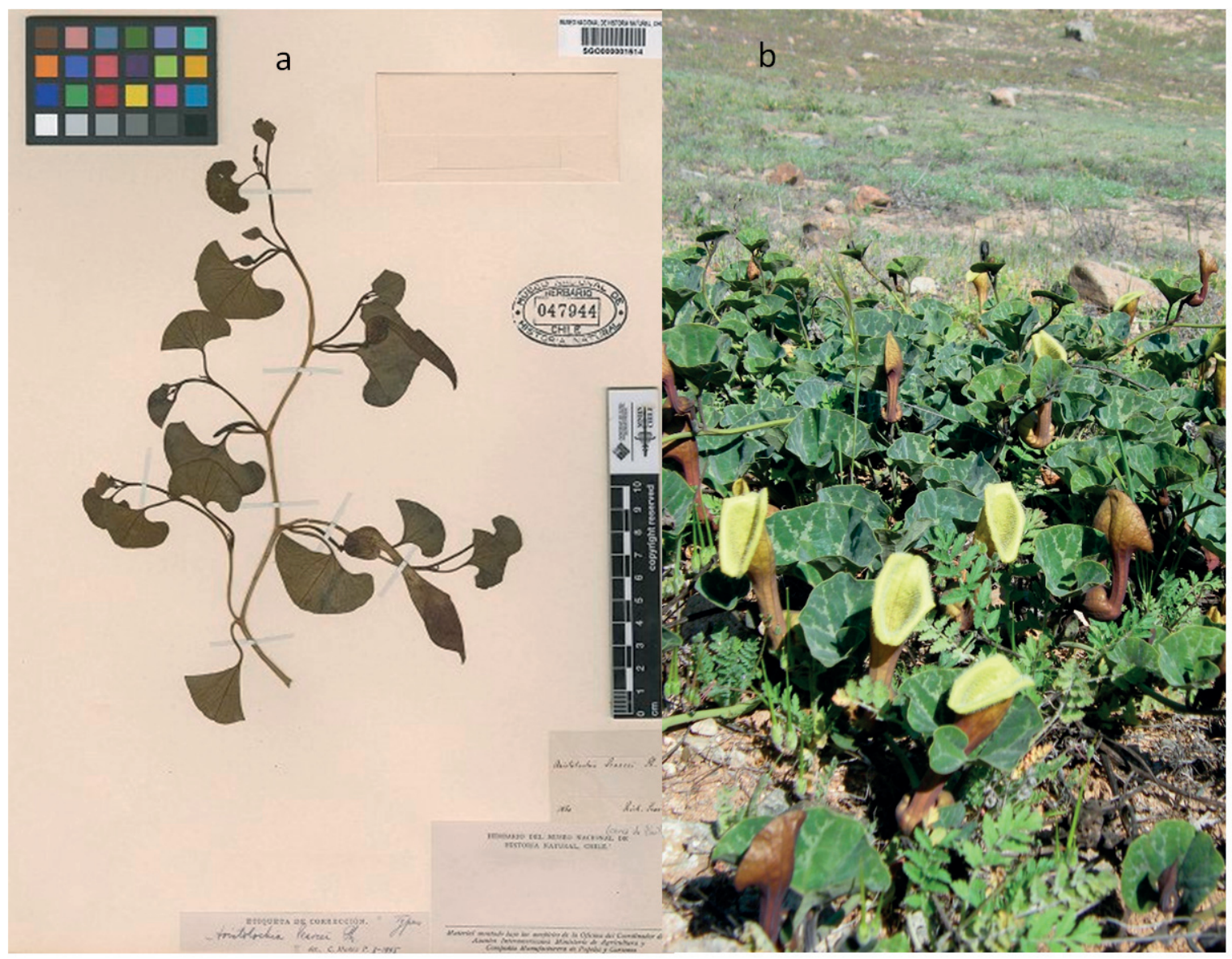

Figura 1. Aristolochia pearcei (a) lectotipo, (b) planta en hábitat, La Higuera, provincia del Elqui, Región de Coquimbo (Fotografías: (a) http://plants.jstor.org, (b) L. Faúndez).

Figure 1. Aristolochia pearcei. (a) lectotype, (b) plant habitat, La Higuera, provincia del Elqui, Región de Coquimbo (Photos: (a) http:// plants.jstor.org, (b) L. Faúndez). 
DistribuCión Y hÁBITAT. Especie endémica de las regiones de Atacama (provincia del Huasco) y de Coquimbo (provincias de Elqui y de Limarí), principalmente en la precordillera andina y en sistemas montañosos que alcanzan a la Cordillera de la Costa entre los ríos Huasco y Elqui. Crece asociada a vegetación del tipo matorral de Balbisia peduncularis (Lindl.) D. Don. y Bahia ambrosioides Lag. que incluyen cactáceas arborescentes como Eulychnia acida Phil. var. acida y Eulychnia breviflora Phil. var. breviflora, esta última sólo en la zona costera. Este tipo de asociación vegetal de acuerdo con el esquema nacional propuesto por Gajardo (1994), se encuentra en las formaciones del Matorral Estepario Interior y Matorral Estepario Costero (Región del Matorral y del Bosque Esclerófilo), y las formaciones del Desierto Florido de las Serranías y del Desierto Costero del Huasco en su porción sur (Región del Desierto).

Materiales adicionales: CHILE, Atacama, provincia de Huasco, Freirina, Totoral, 90 m; 30-IX-1952, M. Ricardi 2323 (CONC 12978); Cachiyuyo, 900 m, 10-X-1958, M. Ricardi 4890 y C. Marticorena 1275 (CONC 25685);
Vallenar, Quebrada $10 \mathrm{~km}$ al sur, camino a La Serena, 620 m, 12-VI-1956, M. Ricardi 3907 y C. Marticorena (CONC 24026); Domeyko, Quebrada de Algarrobal interior, 500600 m, 16-XI-1956, M. Ricardi 4006 y C. Marticorena (CONC 24120); Coquimbo, provincia de Elqui, Incahuasi. $20 \mathrm{~km}$ al sur, $300 \mathrm{~m}, 10-\mathrm{X}-1958$, M. Ricardi 4894 y C. Marticorena 1279 (CONC 25689); Andacollo, 1000 m, 11X-1958, M. Ricardi 4932 y C. Marticorena 1317 (CONC 25728); Carretera Panamericana, cerros frente al Tofo, 500 m, 14-X-1958, Marticorena y Matthei 197 (CONC 28153); Llanos de La Higuera, frente a El Tofo, 430 m, 16-IX-1965, G. Gleisner 25 (CONC 34499); El Tofo, 550 m, 24-II-1965, M. Ricardi 5262 (CONC 29985); provincia de Limarí, Ovalle, El Reloj, 500 m , 08-IX-1949, C. Jiles 1320 (CONC 30518).

Aristolochia bridgesii (Klotzsch) Duch., in DC. Prodr. 15(1): 463. 1864. Howardia bridgesii Klotzsch, Monatsber. Königl. Preuss. Akad. Wiss. Berlin: 618. 1859. TIPO: Colchagua, Chile, T. Bridges s/n (lectotipo K 323645! aquí designado). Figura 2.

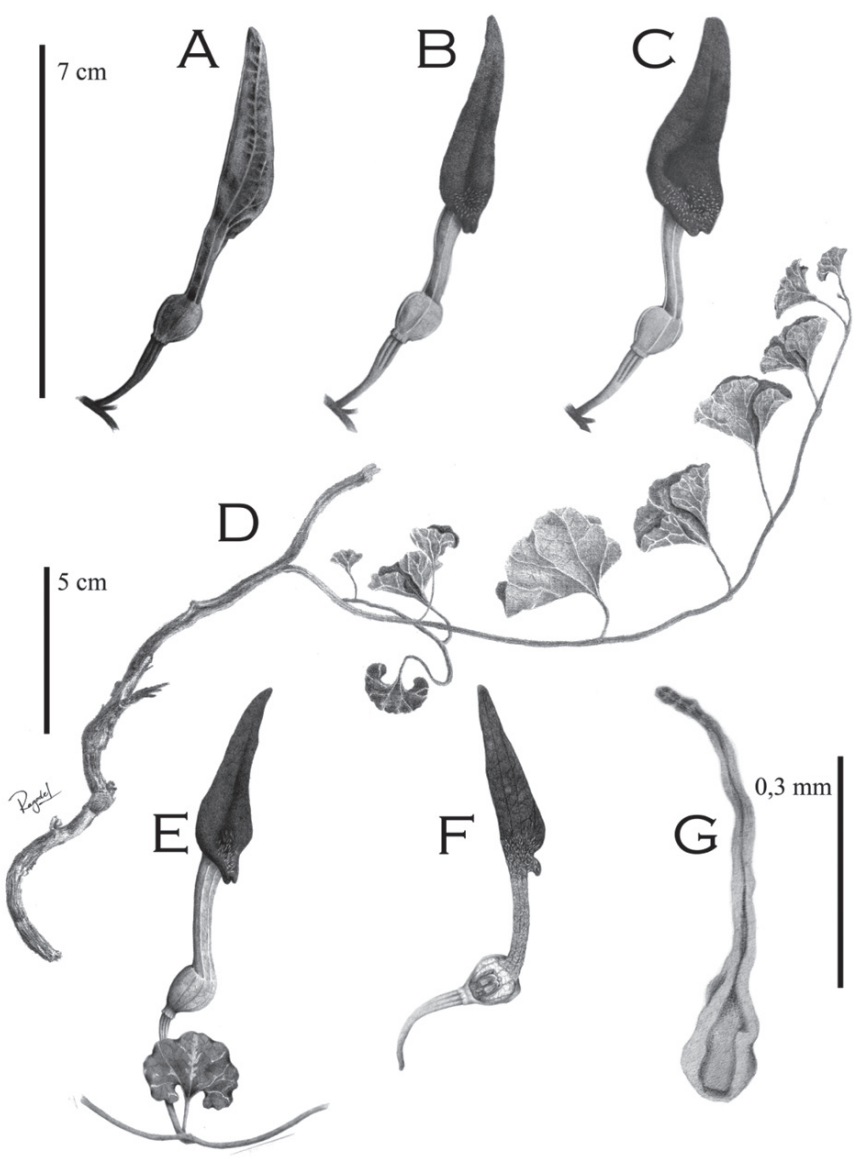

Figura 2. Aristolochia bridgesii. (a, b y c) desarrollo floral, desde botón hasta antesis, (d) hábito, (e) rama florífera, (f) detalle interno de la flor, (g) pelo interno del perigonio. Dibujos originales de Rafael González de CONC 175573.

FiguRE 2. Aristolochia bridgesii. (a, b and c) floral development, from button until anthesis, (d) habit, (e) floriferous branch, (f) internal detail of the flower, (g) perianths internal hair. Original drawings of Rafael González of CONC 175573. 
Hierba perenne de ramas prostradas, procumbentes, de base semiherbácea, surgiendo desde un xilopodio, con entrenudos de 3-6 cm. Hojas alternas, lámina reniforme, margen cartilaginoso entero a levemente irregular, de 2,5$5,5 \mathrm{~cm}$ de ancho, glabra en la cara adaxial y pubérula en la abaxial, con peciolos de 2-4 cm de longitud. Flores de color marrón oscuro por dentro y algo más claras por fuera, solitarias y axilares. Pedúnculo floral y ovario de $2,5-3 \mathrm{~cm}$ de longitud, glabros. Perigonio glabro por fuera; internamente el utrículo, tubo, fauces y base del labio cubiertos por pelos blancos esparcidos; el resto de la superficie del labio completamente glabro. Utrículo de 1-1,2 cm de longitud $\mathrm{x}$ 0,7-1 cm de ancho, dispuesto en ángulo recto a algo obtuso en relación con el tubo. Tubo de 2,5-3 cm de longitud x 0,5 $\mathrm{cm}$ de ancho, cilíndrico. Labio originado de la parte anterior de las fauces y circundándolas, la base levemente hendida y revoluta, extendiéndose posteriormente de forma deltoide a triangular con el ápice agudo, de igual longitud o mayor que el tubo, recto o vertical, siguiendo la proyección del tubo, a un poco arqueado en la antesis. Ginostemio 6-lobado, de $5 \mathrm{~mm}$ de longitud, anteras de $2 \mathrm{~mm}$ de longitud, lóbulos del ginostemio de cerca de $2 \mathrm{~mm}$ de longitud y ápices algo incurvos. Fruto una pseudocápsula oblonga, hexagonal, de 6-9 $\mathrm{cm}$ de longitud, incluido el pedúnculo y $2 \mathrm{~cm}$ de ancho, glabra, con estrías transversales poco evidentes. Semillas ligeramente cóncavas y negruzcas en su parte dorsal y convexas en la ventral, obcordado-triangulares, de aproximadamente $5 \times 3 \mathrm{~mm}$, lustrosas, cubiertas en los márgenes y en el ápice por escamas pequeñas de color marrón claro, con la nervadura central ventral prominente.
Materiales adicionales: CHILE, Región Metropolitana, Prov. Maipo, Cuesta El Cepillo, Rangue, camino a Cholqui, 600 m, XI-1990, Faúndez s.n. (CONC 175573, SGO 161331); Altos de Cantillana, $580 \mathrm{~m}, 30-\mathrm{X}-2010$, Moeller s.n. (AGUCH 65887); San Francisco de Aculeo, 350 m, 29-X-1976, Montero 10246 (CONC 88775); Prov. Melipilla, Cuesta Los Guindos, 290 m, XII-1987, Faúndez s.n. (AGUCH 66122); Curacaví, 1898?, T. Bridges s/n (BM 993831). Región del Libertador General Bernardo O'Higgins, Prov. Colchagua, El Naranjal, 328 m, 3-X2010, Ibarra s.n. (AGUCH 66009); Rinconada El Tambo, Lomas del Pangalillo, 409 m, 19-XI-2005, García et al. 1963 (AGUCH 66123). Región del Maule, Colchagua, 1844?, T. Bridges s/n (BM 617504).

DistRIBUCIÓN Y HÁBITAT. Especie conocida hasta la fecha en las laderas bajas de los cerros de la vertiente norte del sistema de Altos de Cantillana en la Región Metropolitana y en las laderas del cordón de cerros al sur de San Vicente de TaguaTagua en la Región de O'Higgins. Probablemente también en las cercanías de Curicó en la Región del Maule. Se asocia a la vegetación de tipo matorral arborescente de Lithrea caustica (Molina) Hook. \& Arn. con árboles remanentes de Quillaja saponaria Molina, la que, según el esquema nacional de vegetación propuesto por Gajardo (1994), corresponde a la formación del Bosque Esclerófilo Costero (región del Matorral y del Bosque Esclerófilo).

De este modo, el género Aristolochia en Chile se encuentra representado hasta la fecha por tres entidades, las cuales se pueden diferenciar según la siguiente clave.

1. Tubo cilíndrico. Labio vertical, de largo igual o mayor al tubo, pubescente solo en la base y fauces. Hojas glaucas, glabras en la cara adaxial y pubérulas en la abaxial..

1'. Tubo infundibuliforme. Labio oblicuo, más corto que el tubo, completamente pubescente. Hojas verdes, manchadas de tonos blancos.

2. Labio de $1 / 3$ a $1 / 2$ del largo del tubo. Hojas glabras en la cara adaxial y pubérulas en la abaxial ......

2'. Labio de $1 / 2$ a $3 / 4$ del largo del tubo. Hojas pubérulas en ambas caras

\section{CONCLUSIÓN}

Se concluye que Aristolochia bridgesii y Aristolochia pearcei corresponden a especies distintas y de distribución alopátrica, separadas por aproximadamente $300 \mathrm{~km}$ de distancia, habitando condiciones climáticas y vegetacionales distintas, siendo $A$. bridgesii la especie conocida de distribución más sur del género en Chile y $A$. pearcei la especie con distribución norte que alcanza las mayores elevaciones en el cordón andino.

Con la presente validación, el género Aristolochia queda representado en Chile por tres especies endémicas de la región mediterránea, desde el sur del Desierto de Atacama hasta la zona central de los Bosques esclerófilos.

\section{AGRADECIMIENTOS}

A Alicia Marticorena por su ayuda en CONC y por sus sugerencias al manuscrito, las que ayudaron a clarificar sustancialmente su contenido; a Gloria Rojas y Jimena Arriagada por las facilidades para estudiar el tipo de $A$. pearcei en SGO. A Aira Faúndez y Rodrigo Flores por su ayuda en el herbario AGUCH y en el trabajo en el herbario CONC. A Rafael González por la elaboración de las ilustraciones de $A$. bridgesii y a Pablo Sandoval por la diagramación de ellas. Finalmente agradecemos a Rosa A. Scherson por la traducción del resumen. Un especial reconocimiento a "The Global Plant Iniciative"(plants. jstore.org) por el importante aporte que significan las facilidades que otorga al trabajo taxonómico. 


\section{LITERATURA CITADA}

Duchartre, P. 1864. Ordo CLXXV. Aristolochiaceae. In: De Candolle, Prodromus Systematis Naturalis Regni Vegetabilis 15(1): 421-498.

Gajardo, R. 1994. La vegetación natural de Chile. Clasificación y distribución geográfica. Editorial Universitaria. Santiago, Chile. 165 pp.

JoHnston, I.M. 1928. The botanical activities of Thomas Bridges. Contributions from the Gray Herbarium of Harvard University 81: 98-106.

KLotzsch, J.F. 1859. Nachtrag. Die Aristolochiaceae des Berliner Herbariums. Monatsberichte der Königlich Preussischen Akademie der Wissenschaften zu Berlin. 571-635.

Marticorena, C. 1990. Contribución a la estadística de la flora vascular de Chile. Gayana Botánica 47: 85-113.

Marticorena, C. \& M. Quezada, 1985. Catálogo de la flora vascular de Chile. Gayana Botánica 42: 1-57.

Muñoz-Pizarro, C. 1960. Las especies de plantas descritas por R. A. Philippi en el siglo XIX. Ediciones de la Universidad de Chile, Santiago. 189 pp.
PhILIPPI, R.A. 1861. Observaciones botánicas sobre algunas plantas recojidas en Chile por los señores don Ricardo Pearce i don Jerman Volckmann. Comunicación del señor Philippi a la Facultad de Ciencias Físicas. Anales de la Universidad de Chile 18: 43-69.

PhilipPI, R.A. 1865. Plantarum novarum Chilensium. Centuriae inclusis quibusdam Mendocinis et Patagonicis. Linnaea 33: $1-308$.

PhiLIPPI, R.A. 1895. Plantas nuevas chilenas de las familias que corresponden al tomo V de la obra de Gay: (continuación). Anales de la Universidad de Chile 91: 487-526.

RuIz, E. 2001. Aristolochiaceae. En: C. Marticorena \& R. Rodríguez (eds.), Flora de Chile Vol. 2, pp. 33-34. Ediciones Universidad de Concepción, Concepción, Chile.

Zuloaga, F.O., O. Morrone \& M.J. Belgrano (eds.). 2008. Catálogo de las plantas vasculares del Cono Sur de América (Argentina, sur de Brasil, Chile, Paraguay y Uruguay). Volumen 2. Dicotyledonae: Acanthaceae Fabaceae (Abarema - Schizolobium). Monographs in Systematic Botany from the Missouri Botanical Garden 107: 985-2286.

Recibido: 27.07.12

Aceptado: 19.08 .15 